\title{
Intrauterine Contraceptive Device, Simple Yet Still A Dilemma
}

\author{
Myat San Yi*, Lim Yee Cherng, Mi Mi Khaing and Rafiae Amin \\ Faculty of Medicine and Health Sciences, Department of Obstetrics and Gynecology, Malaysia \\ Received: 㘹July 25, 2018; Published: 眥 August 01, 2018 \\ *Corresponding author: Myat San Yi, Faculty of Medicine and Health Sciences, Department of Obstetrics and Gynecology, Malaysia
}

\section{Introduction}

Intrauterine contraceptive device (IUCD) is one of the contraceptive methods and its efficacy is as high as $90 \%$ [1]. Increasing use of this device has led to an increase in its related complications. These complications include infection (1\%), uterine perforation $(0.1 \%)$, expulsion of device $(5 \%)$, failure to prevent pregnancy as well as ectopic pregnancy (0.5-1\%), menstrual problems like menorrhagia or dysmenorrhea, migration into the pelvis cavity(misplacements) (5\%) and the frequent clinical problem is the lost tail or loss of the filament at the external cervical os [2]. There were so many studies about IUCD and its sequelae. The commonest and most attractive area is misplacement or migration and loss of IUCD thread. Clinicians from all over the world proved that hysteroscope is the best option to find the lost device in the uterine cavity. There were studies recommending usage of hysteroscopy for embedded or displaced IUCD. A study by Zuan Chong Feng et al. mentioned that hysteroscopy with an ultrasound B-scan is of great value not only for precise location but also for its removal under direct vision, particularly in the management of patients with broken and/or embedded IUCD pieces [3]. A study by Dwyer and James revealed that the incidence of difficulties associated with IUCD removal may occur in up to $9 \%$ of follow-up visits of women who have been fitted with IUCD [4]. According to the evidence, incidence of intrauterine device perforation is 0.87 per 1000 insertions [5]. (Ofer Markovitch et al.) Most perforations occur at the time of insertion and the risk is increased in the 4-8 weeks postpartum. When the string is found to be missing, pregnancy must be excluded, and the endometrial cavity explored. Ultrasonography can often determine if the IUCD is in the uterus; most IUCDs that perforate the uterus are often found in the pelvis $[5]$.

There are several studies looking into factors influencing uterine perforation during insertion. These factors consist of the insertion during post-partum or lactation period, the force used during insertion and the experience of the operator. There are 3 types of perforation classified according to the compartments involved:
A. Compartment $1=$ uterine cavity
B. Compartment 2= Myometrium
C. Compartment $3=$ peritoneal cavity [6]

Thus, in

Type 1-2 Partial perforation, the IUD is partially in the uterine cavity and myometrium

Type 2 Myometrium only

Type 2-3 Myometrium and peritoneal cavity

Uterine perforation and deeply embedded intrauterine device require exact determination of its location to ensure safe and smooth retrieval. One study from Radiology Journal suggested doing hysterography as it offered the most precise diagnostic information. With the advent of 3D Ultrasound, the combination of ultrasound and hysteroscopy are well-recognized effective treatment option [7]. For extrauterine misplaced IUCD, surgical removal is recommended by most of the clinicians as there is a putative risk of adhesion formation or damage to the nearby structures such as intestines or bladder [5]. However, there are limited studies regarding embedded IUCDs; although there is a role of hysteroscopy for its removal. There are conflicting evidences in different literatures regarding management of cases of embedded IUCD [8]. The management differs in either to remove the IUCD by using resectoscope or leaving in uterine wall although there is a possibility of migration into the pelvic cavity and abnormal uterine bleeding with infection. A study by Zuan Chong Feng et al indicated one out of 274 cases in their study remained a small piece in the uterine wall due to breakage of IUCD during extraction but, the study did not highlight the outcome of leaving behind small pieces. There will be concerns about the copper used in the device. When biological materials come into contact with copper, it is corroded and the compounds that are formed can produce irritation and other reactions like adhesion. However, as far as we learned, there is no clear evidence that harm is actually done. 
The following case report is the common complication of long-term use of IUCD for 20 years resulting in the breakage of arms of embedded IUCD during retrieval. The aim of this case report is to share the experience that even though one follows the recommended procedure, there is a possibility of failure to achieve the expected goal. In this case, one has to reconsider what should be the best interests of the patient. Clinical judgement between the risks (morbidity) and benefits (expected outcome) of the patient under the ethical consideration plays the most vital role. Madam B, 58 years old, Para 5, postmenopausal housewife, was admitted to our hospital with the complaint of failure to remove the IUCD which was inserted 20 years ago. She has Hypertension on Tablet Amlodipine $5 \mathrm{mg}$ OD and Diabetes Mellitus on Tablet Metformin $750 \mathrm{mg}$ BD under medical clinic follow-up. She never had any surgery before. She was non-smoker and non-alcohol consumer. All her children were delivered vaginally without complications and she decided to be inserted with intrauterine contraceptive device (copper) after the birth of her last child who is now 20 years old. She recalled that time everything went smoothly. After 3 years, she went for clinic follow-up in rural center where she was advised to use the oral contraceptive pills, but, she deferred for IUCD removal. She was a compliant pill-user for 17 years. The IUCD was forgotten for the last 17 years, until September 2017 during a conversation with her family, her daughter learned about it and asked her to be seen by private doctor to remove the device. Unfortunately, it was stuck, and the thread snapped. She was referred to the hospital and during examination, the attending doctor saw a plastic rod at the cervical os. It looked like the body of the intrauterine device with some copper coils and it was around $1.5 \mathrm{~cm}$ long. He was a wellqualified doctor who was cautious enough to do a transabdominal ultrasound before proceeding with removal in the treatment room. The following finding was noted during scan- Axial uterus, the two segments of device were seen with one segment at the cervical canal and the other in the endometrial cavity (Figure 1).

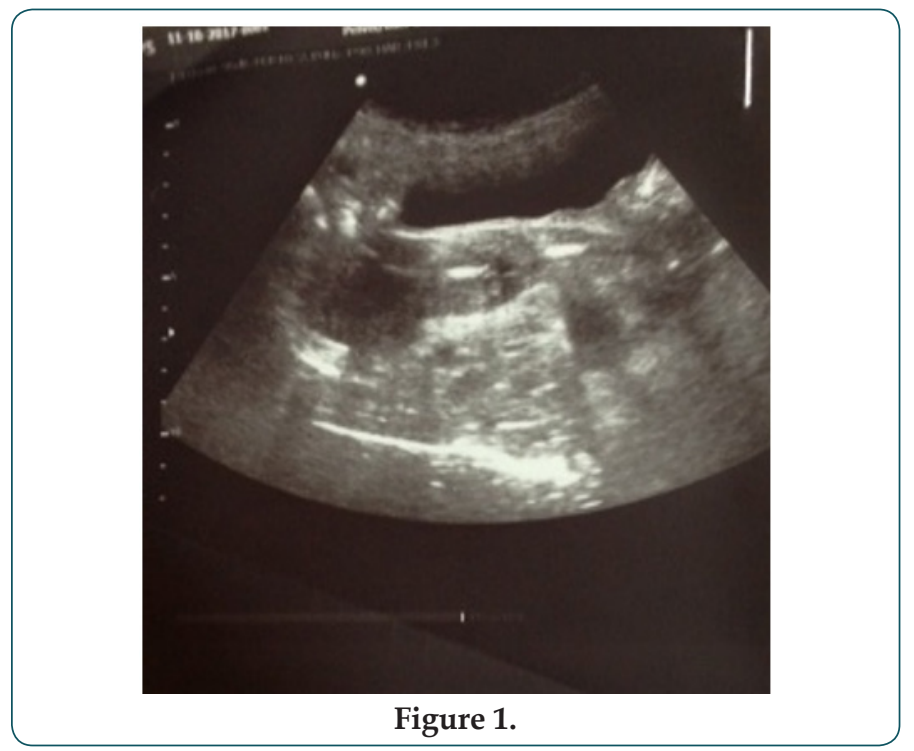

After the ultrasound, he gently removed the device with a sponge forceps from the endocervical canal, however he was unsuccessful to remove the device completely. As the hysteroscopic [9] removal of IUCD is a standard recommended procedure, he arranged to remove the intrauterine device in the uterine cavity in the operation theatre after thorough explanation and taking informed consent.

During the procedure, the following findings were noted:

I. Vaginal canal and Cervix- healthy, Uterus was small, the endometrium was atrophic. Both ostia were seen clearly.

II. The IUCD was noted easily as a white plastic rod close to the endocervical canal and it was $1.5 \mathrm{~cm}$ in length.To the surgeon's dismay, he could not locate the arms of the intrauterine device and the rod was easily and cautiously removed $[10,11]$.

Total length for the segments removed was $3 \mathrm{~cm}(1.5 \mathrm{~cm}$ at triage area and $1.5 \mathrm{~cm}$ intraoperatively). After the procedure, the case was discussed with the consultant and decided for abdominal X-ray which showed two small radiopaque shadows. The possibility of two plastic foreign body (likely arms of IUCD) embedded in the uterine wall was decided (Figure 2).

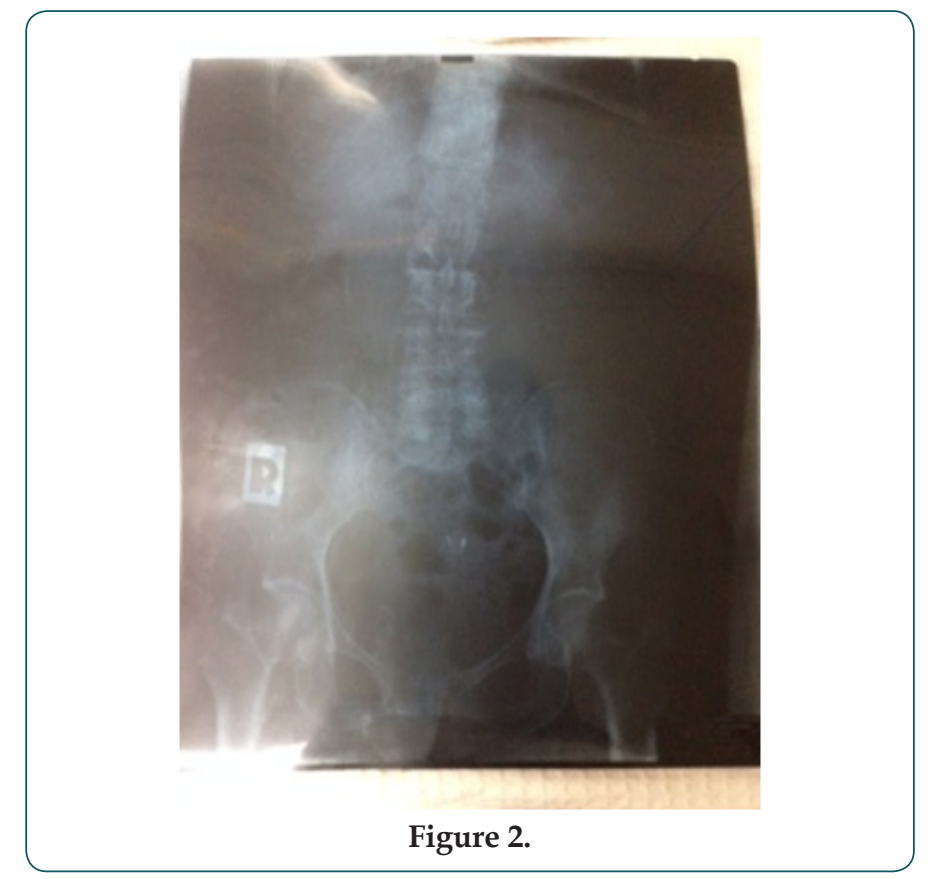

De-briefing on the current condition was made. Two options with either close monitoring or resectoscopic removal was discussed with the patient including pros and cons in terms of bleeding from resection and other rare complications like injury to nearby structures were highlighted. She made a choice for close monitoring and will turn up immediately if any symptoms arise in the future. She also agreed to repeat the ultrasound scan to take note of the displacement and watch out for any signs and symptoms of infection especially endometritis or pelvic inflammatory disease. Although there is a risk of perforation and migration of IUCD remnants in future, we emphasized that the retained foreign bodies will remained embedded. We honestly counselled the patient that 
these were our opinion based on our clinical experience. In this case report, instead of removing the remnants of the IUCD, we decided to leave behind and kept her under close monitoring. We planned to repeat the ultrasound by consultant during her next visit to monitor whether the remnants become displaced or not. We will review her symptoms in every visit as infection is our sole concern. In conclusion, this case reflects the well-known complication of long-time IUCD user. Despite the standardized treatment in expert hands, the dilemma still exists in deciding the best management option. To the best of our knowledge, embedded small pieces (arms) were only made of plastic, not of copper and we are relieved assuming that it will not be harmful to the patient. One may still argue "Your assumption is not proved in the scientific way" and in this case, we will respect their point of view. However, for a clinician, what matters most is as long as our patient is safe and sound, we should accept that our management is justifiable. (Benefit should be more than risk) We would like to end our case report with a quote from Grantly Dick-Read (1890 -1959), a British obstetrician and a leading advocate of natural birth, quoted as saying "clinician should use the method that gives the best and safest result from all points of view until something better is discovered."

\section{References}

1. Katherine Rivlin, Carolyn Westhoff $\left(7^{\text {th }}\right.$ edn.) Family Planning, Comprehensive Gynecology. 13, 237-257.
2. (2018) Intrauterine contraceptive device (IUD, IUCD). Ministry of Health.

3. Zuan Chong Feng, Yong Peng Shi, Xue Zhe Wu, Su Ping Liu (2001) The application of hyseroscopy in diagnosis and treatment of missing intrauterine devices.

4. Dwyer NA, James DK (1991) Removal of an embedded IUD using the resectoscope. British Journal of Family Planning 16(4): 145.

5. Ofer Markovitch, ZviKlein, Yariv Gidoni, Michael Holzinger, Yoram Beyth (2002) Extrauterine mislocated IUD: is surgical removal mandatory? Contraception 66(2): 105-108.

6. (1985) European Journal of Obstetrics and Gynaecology and Reproductive biology 19(1): 37- 41.

7. Rosenblatt R, Zakin D, Stern WZ, Kutcher R (1985) Uterine perforation and embedding by intrauterine device: evaluation by US and hysterography. Radiology 157(3): 765-770.

8. David Zakin, Wilhelm Z Stern, Ruth Rosenblatt (1982) Perforated and embedded intrauterine devices. JAMA 247(15): 2144-2146.

9. Shubha Sagar Trivedi, Madhu Goel, Sandhi Jain (2000) Hysteroscopic management of intrauterine devices with lost strings. 26(4): 229-230.

10. David K Turok, Shawn E Gurtcheff, Keri Gibson, Erin Handley, Sara Simonsen, et al. Operative management of intrauterine device complications: a case series report. Contraception82(4): 354-357.

11. Siegler AM, Kemmann E (1976) Location and removal of misplaced or embedded intrauterine devices by hysteroscopy. J Reproductive Medicine 16: 139-144.

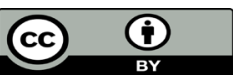

This work is licensed under Creative Commons Attribution 4.0 License

To Submit Your Article Click Here: Submit Article

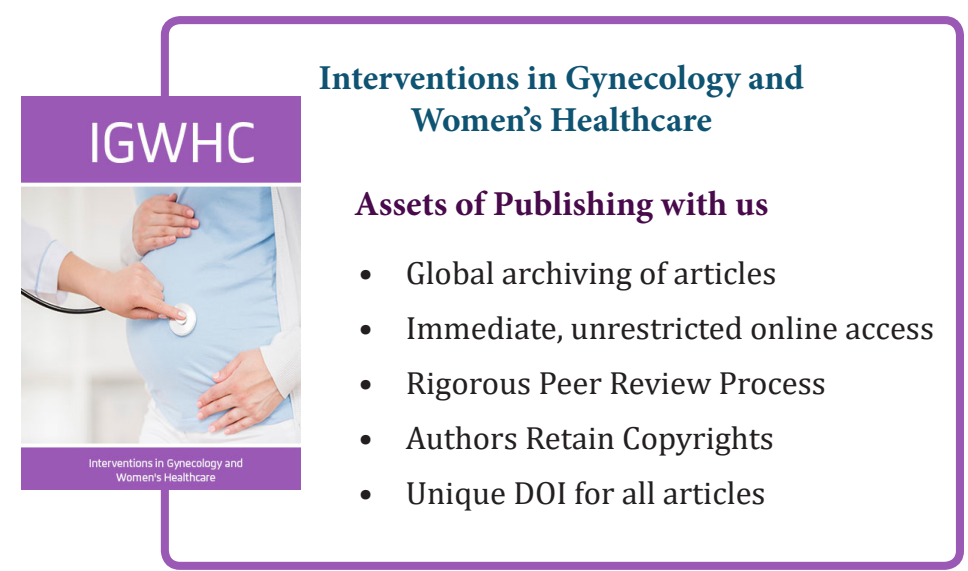

